showed necrosis of the pancreatic body and tail. On the eighth day of the attack he became hypertensive, unconscious, and unresponsive, with increasing fever despite antibiotics. A 1.8 litre plasma exchange was performed as in case 1. Confusion cleared rapidly, vital signs and oxygenation improved, fever decreased (figure), and the APACHE II score fell from 17 to 9. Next day the necrotic pancreatic tail and body were debrided at laparotomy and irrigating drains inserted. He made a complete recovery and was discharged 38 days later.

\section{Comment}

Many patients with fulminant acute pancreatitis have pancreatic necrosis, and survival depends on debridement of necrotic or infected tissue. If operation is undertaken during the initial week survival is unlikely, ${ }^{+}$and the outlook is similarly bleak if multiorgan failure becomes established.' There is a logical role for treatments that allow debridement to be undertaken at a stage when recovery can be expected. Plasmapheresis scavenges various circulating toxins, including protease- $\alpha_{2}$ macroglobulin complexes. Our findings are preliminary, but the similarity of response was impressive, and in both cases there was a large reduction in the APACHE II score with the expectation of an increased probability of survival. Plasmapheresis appeared to induce more pronounced changes in the course of acute pancreatitis than other treatments that we have employed. Further evaluation is needed, preferably in patients who have not developed the manifestations of overt multiorgan failure and in the setting of a protocol which includes surgical debridement, when appropriate, on the basis of objective criteria including CT angiography and precise physiological monitoring.

1 Larvin M, Switala SF, McMahon MJ. Impaired clearance of circulating protease-antiprotease complexes during acute pancreatitis: an importan proteasc Sniprome

2 Ranson JH, Rifkind KM, Roses DF, Eng K, Spencer FC. Prognostic signs and the role of operative management in acute pancreatitis. Surg Gynecol Obste 1974:139:69-81.

3 Knaus WA, Draper EA, Wagner DP, Zimmermann JE. APACHE-II: a severity of disease classification system. Crit Care Med 1985;13:818-29.

+ Smadja C, Bismuth H. Pancreatic debridement in acute necrotising pancreatitis an obsolete procedure? Br $\mathcal{F}$ Surg 1986;73:408-10.

5 Glazer G, Dudley HAF. The surgical management of pancreatic abscess. Ann $R$ Coll Surg Engl 1987;69:249-50.

(Acçpted 9 Mư 1988)

\title{
Human leucocyte antigen in a Chinese family with thyrotoxic periodic paralysis in Singapore
}

\section{F Sum, A C K Fok, K T Tan, S H Chan, J S Cheah}

University Department of Medicine, Singapore General Hospital, Singapore 0316, Republic of Singapore CF Sum, MRCP, registrar A C K Fok, MRCP, registrar K T Tan, MRCP, registrar J S Cheah, FRACP, professor of medicine

\section{Department of}

Microbiology, National University of Singapore and World Health Organisation Immunology Centre, Singapore

S H Chan, FRCPA, professor of microbiology

Correspondence and requests for reprints to: DrSum.
Periodic paralysis is a dramatic complication in some patients who have thyrotoxicosis (Graves' disease). It occurs more commonly in thyrotoxic men of Mongoloid descent.

The familial occurrence of thyrotoxic periodic paralysis is, however, infrequent. To date there have been only a few reports of familial thyrotoxic periodic paralysis. Most reports have been based on a positive family history elicited during questioning of the patient. We report the first, to our knowledge, documented occurrence of familial thyrotoxic periodic paralysis in a Chinese family in Singapore together with the human leucocyte antigen genotypes of the family members.

\section{Case report}

A 31 year old Chinese man was admitted with characteristic clinical and biochemical features of hypokalaemic thyrotoxic periodic paralysis. His thyrotrophin receptor antibody concentration was

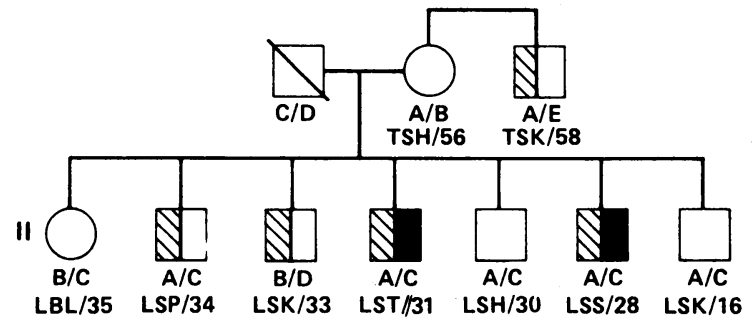

Human leucocyte antigen haplotypes:

$A=A 9 \quad$ BW22 DR9 DRw53 DQw3

$B=A 2 \quad \cdots . . . \quad$ DR9 DRw53 DOw3

$C=A 11 \quad B 16 \quad$ DR2 $\quad$ DRw52 DQw

$D=A 26 \quad B 15$ DR5 DRw52 DQw1

$\mathrm{E}=\mathrm{A} 11$ BW46 DR2 ….. DQW1

Family pedigree and human leucocyte antigen genotypes
$195 \mathrm{IU} / 1$ (normal < $10 \mathrm{IU} / \mathrm{l})$. He was treated with oral potassium supplements together with propranolol and carbimazole. The next day he had completely regained his muscular power. The case records of all his immediate family members who had thyrotoxicosis or thyrotoxic periodic paralysis, including a thyrotoxic maternal uncle, were recalled and studied. Subsequently the human leucocyte antigen type of all the immediate family members and maternal uncle was tested with the National Institutes of Health lymphocyte microcytotoxicity method.' The figure shows the pedigree and human leucocyte antigen genotypes of the family.

\section{Comment}

Lately there has been much interest in the genetics of thyrotoxicosis and thyrotoxic periodic paralysis. Chan et al reported an association between human leucocyte antigen Bw46 and DR9 and thyrotoxicosis in Chinese patients. ${ }^{2}$ Those members of the family whom we studied who had documented thyrotoxicosis (with or without periodic paralysis) were haplotype A or B, each of which contains the alleles DR9, DRw53, and DQw3.

In addition, Yeo et al showed that the haplotypes A2 Bw22 and Aw19 B17 were associated with thyrotoxic periodic paralysis among Chinese patients in Singapore. ${ }^{3}$ It was reported that Bw22 and B17 were in linkage disequilibrium with $\mathrm{A} 2$ and Aw19, respectively. In another study Cheah concluded that it was the alleles Bw22 and B17 that carried an increased relative risk for thyrotoxic periodic paralysis. ${ }^{4} \mathrm{He}$ also observed that the haplotype A2 Bw22 occurred only in Chinese thyrotoxic patients who had periodic paralysis and was absent in Chinese thyrotoxic patients who did not have periodic paralysis.

In the family that we studied, besides the two siblings who had thyrotoxic periodic paralysis, three other siblings were haplotype $A$ thus carrying the allele Bw22. It is tempting to speculate that thyrotoxic periodic paralysis may occur in these three siblings should the thyrotoxic state recur.

Familial thyrotoxicosis occurs more commonly than familial thyrotoxic periodic paralysis. Several studies of human leucocyte antigen have been performed in such families. On reviewing the cumulative results of studies of white and Chinese subjects Tian et al noted that of 36 different families that had multiple cases of 
thyrotoxicosis, two thirds of the affected sibling pairs shared both parental haplotypes. It was postulated that both parental haplotypes were important for the development of thyrotoxicosis. In other words the human leucocyte antigen gene contributing to the development of thyrotoxicosis may be inherited in a recessive fashion.'

Separate alleles (Bw22 and B17) have been suggested as markers for thyrotoxic periodic paralysis. It remains unclear whether the mode of inheritance of thyrotoxic periodic paralysis is similar to that of thyrotoxicosis per se. This family provided us with a unique opportunity to glimpse the genetic mechanisms occurring in thyrotoxic periodic paralysis. The occurrence of identical haplotypes in the sibling pair who had

\section{Notification of tuberculosis: Can the pathologist help?}

\section{B L Bradley, K M Kerr, A G Leitch, D Lamb}

Royal Victoria Dispensary for Diseases of the Chest, Edinburgh

B L Bradley, MRCP, registrar A G Leitch, $\mathrm{FRCP}$, consultant physician

Department of Pathology, University of Edinburgh K M Kerr, MB, senior registrar D Lamb, FRCPATH, reader

Correspondence to: $\mathrm{Dr}$ A G Leitch, Chest Unit, City Hospital, Edinburgh thyrotoxic periodic paralysis suggests that the mode of inheritance of thyrotoxic periodic paralysis and thyrotoxicosis may indeed be similar.

1 Mittal KK, Mickey MR, Singal DP, Terasaki PI. Serotyping for homotransplantation 18: refinement of microdroplet lymphocyte cytotoxicity test. Transplantation 1968;6:913-27.

2 Chan SH, Yeo PPB, Tan SH, Wee GB, Lui KF, Cheah JS. Bw46 and DRw9 associations in Singaporean Chinese Graves' disease. In: Aizawa M, ed. HLA in Asia-Oceania. Japan: Hokkaido University Press, 1986:771-3.

3 Yeo PPB, Chan SH, Lui KF, Wee GB, Lim P, Cheah JS. HLA and thyrotoxic periodic paralysis. Br Med $\mathcal{f} 1978$;ii:930.

4 Cheah JS. Thyrotoxic periodic paralysis in Singapore. In: Cheah JS, Lim P, Tambyah JA, et al, eds. Proceedings of the Sixth Asia and Oceania Congress of Endocrinologv, Singapore 1978;1:282-99.

5 Tian D, Mao TS, Yang SH, et al. HLA haplotypes sharing in multiple thyrotoxicosis families. In: Aizawa M, ed. HLA in Asia-Oceania. Japan: Hokkaido University Press, 1986:764-7.

(Accepted 21 April 1988)
The incidence of tuberculosis in Great Britain has declined steadily over recent decades, the trend being confirmed by statutory notification data. ' : In 1986 over 6000 cases of tuberculosis were notified but this figure may be too low, for ambiguities and inaccuracies are recognised to exist in the notification system in England and Wales. ${ }^{3}$ Data for the United States show that $37 \%$ of cases go unreported. ${ }^{+}$

For many years in Scotland it has been the practice to notify all positive bacteriological results to cover possible failures in notification by clinicians. We wondered whether a similar procedure of notification for positive pathological biopsy specimens would further improve notification practices. We have therefore identified pathological diagnoses of tuberculosis over four years, examined their characteristics, and related the findings to notification data.

\section{Methods and results}

All pathology reports issued from the University Department of Pathology, Edinburgh, in which tuberculosis was listed as the primary diagnosis or in the differential diagnosis were examined for the years 1981-4. The slides of all cases were reviewed. Patients' case notes were scrutinised for details of diagnosis, treatment, and whether a respiratory physician had been consulted. Cases were checked against the local tuberculosis register for the corresponding years to see whether they had been notified.

Pathology reports were coded as follows: (A) a firm pathological diagnosis of tuberculosis in which acid fast organisms were present in the sections; (B) strongly suggestive or firm diagnosis of tuberculosis made on morphological grounds, though acid fast bacilli were not seen; (C) cases in which a firm diagnosis of inactive, calcified, or healed tuberculosis was made; (D) cases in which tuberculosis was mentioned in the differential diagnosis for confirmation or exclusion on clinical grounds.

Eighty two sets of case notes and pathology reports were examined. Eight sets of case notes could not be traced. Thirty four cases were coded A, $35 \mathrm{~B}$, five C, and eight $\mathrm{D}$

Codes $A$ and $B$-The table gives the age and sex characteristics of the 69 patients coded A or B by the pathologist together with information on the number of notifications and number of consultations with a respiratory physician. Of patients in whom acid fast bacilli were identified, $11(32 \cdot 4 \%)$ were not notified, and of those in whom acid fast bacilli were not seen but a firm pathological diagnosis was made, $15(42.9 \%)$ were not notified. Only eight of the 69 patients were not seen by a respiratory physician. One of the unnotified patients had acid fast bacilli in a sputum smear. Sixty two of the the 69 patients were treated for tuberculosis. Of these 62 patients treated, and therefore considered to have active disease, only two thirds were notified. In this group 50 patients $(81 \%)$ had chemotherapy for the standard six or nine months. Unusual treatment combinations, all based on rifampicin, were employed in 12 patients, on four occasions by chest physicians. Failure to notify was equally common in physicians and surgeons, though the comparatively larger number of pathological diagnoses on material obtained by surgeons $(48 v 21)$ led to a greater number of failed notifications.

Codes $C$ and $D$-None of the 13 patients coded $C$ or $\mathrm{D}$ was notified or treated as a case of tuberculosis.

Age and sex characteristics of patients coded $A$ or $B$ with details of number notified and number seen by respiratory physician

\begin{tabular}{llll}
\hline & Code A & Code B & Total \\
\hline Mean age in years (range) & $53(9-80)$ & $52(11-87)$ & $53(9-87)$ \\
No male & 21 & 16 & 37 \\
No female & 13 & 19 & 32 \\
No notified & 23 & 20 & 43 \\
No(\%) not notified & $11(32 \cdot 4)$ & $15(42 \cdot 9)$ & $26(37 \cdot 7)$ \\
No not seen by respiratory & & \multicolumn{2}{c}{} \\
$\quad$ physician & 4 & \multicolumn{2}{c}{8} \\
Notified & 2 & 1 & 3 \\
Not notified & 2 & 3 & 5 \\
\end{tabular}

\section{Comment}

This study shows that almost $40 \%$ of patients (26/69) with a convincing combined clinical and pathological diagnosis of tuberculosis were not notified, in keeping with findings in the United States. ${ }^{+}$Most failures of notification occurred in surgical wards, though physicians also failed to notify positive pathological diagnoses of tuberculosis. Fortunately, treatment was conventional in most cases and respiratory physicians were consulted in most. Our study suggests that all positive pathological diagnoses of tuberculosis should be notified to the local health board to ensure that notifications reflect the true incidence of disease. This would also ensure that appropriate contact procedures can be instituted.

1 MRC Tuberculosis and Chest Disease Unit. National survey of tuberculosis notifications in England and Wales 1978-9. Br Med J 1980;281:895-9.

2 MRC Tuberculosis and Chest Diseases Unit. National notifications of tuberculosis in England and Wales 1983. Br Med $\mathcal{J}$ 1985;291:658-61.

3 Davies PDO, Darbyshire J, Nunn AJ, el al. Ambiguitics and inaccuracies in the notification system for tuberculosis in England and Wales. Community Med 1981:3:108-18.

Marier R. The reporting of communicable diseases. Am 7 Epidemio 1977; 105:587-90.

5 Capewell SC, Leitch AG. The value of contact procedures for tuberculosis in Edinburgh. Br 7 Dis Chest 1984;78:317-29.

(Accepted 10 Mav 1988) 